\section{A conserved transcriptional regulator is required for RNA-directed DNA methylation and plant development}

\author{
Xin-Jian He, ${ }^{1,7}$ Yi-Feng Hsu, ${ }^{1,2,7}$ Shihua Zhu, ${ }^{3}$ \\ Hai-Liang Liu, ${ }^{1}$ Olga Pontes, ${ }^{4}$ Jianhua $\mathrm{Zhu}^{5}{ }^{5}$ \\ Xinping Cui, ${ }^{6}$ Co-Shine Wang, ${ }^{2}$ \\ and Jian-Kang $\mathrm{Zhu}^{1,8}$
}

\begin{abstract}
${ }^{1}$ Institute for Integrative Genome Biology and Department of Botany and Plant Sciences, University of California at Riverside, Riverside, California 92521, USA; ${ }^{2}$ Graduate Institute of Biotechnology, National Chung Hsing University, Taichung 40227, Taiwan; ${ }^{3}$ College of Science and Technology, Ningbo University, Ningbo 315211, China; ${ }^{4}$ Biology Department, Washington University in St. Louis, St. Louis, Missouri 63130, USA; ${ }^{5}$ Department of Plant Science and Landscape Architecture, University of Maryland, College Park, Maryland 20742, USA; ${ }^{6}$ Department of Statistics, University of California at Riverside, Riverside, California 92521, USA
\end{abstract}

RNA-directed DNA methylation ( $\mathrm{RdDM})$ is a conserved mechanism for epigenetic silencing of transposons and other repetitive elements. We report that the $r d m 4$ (RNA-directed DNA Methylation4) mutation not only impairs RdDM, but also causes pleiotropic developmental defects in Arabidopsis. Both RNA polymerase II (Pol II)- and Pol V-dependent transcripts are affected in the $r d m 4$ mutant. RDM4 encodes a novel protein that is conserved from yeast to humans and interacts with Pol II and Pol V in plants. Our results suggest that RDM4 functions in epigenetic regulation and plant development by serving as a transcriptional regulator for RNA Pol V and Pol II, respectively.

Supplemental material is available at http://www.genesdev.org.

Received August 8, 2009; revised version accepted October 2, 2009.

DNA methylation and histone modifications are important epigenetic silencing mechanisms in eukaryotic cells (Chan et al. 2005; Matzke and Birchler 2005). In plants, DNA methylation is catalyzed by DNA methytransferase MET1, CMT3, and DRM2 (Chan et al. 2005). MET1 and CMT3 mainly function in maintaining DNA hypermethylation at $\mathrm{CG}$ and $\mathrm{CHG}$ sites ( $\mathrm{H}$ is $\mathrm{A}, \mathrm{T}$, or $\mathrm{C})$ during DNA duplication, while the function of DRM2 is involved in de novo DNA methylation at $\mathrm{CHH}$ sites directed by RNA (Chan et al. 2005). RNA-directed DNA methylation (RdDM) was first discovered in plants

[Keywords: RDM4; siRNA; transcriptional gene silencing; Pol II; Pol V] ${ }^{7}$ These authors contributed equally to this work.

${ }^{8}$ Corresponding author.

E-MAIL jian-kang.zhu@ucr.edu; FAX (951) 827-7115.

Article published online ahead of print. Article and publication date are online at http://www.genesdev.org/cgi/doi/10.1101/gad.1851809.
(Wassenegger et al. 1994), and plays important roles in transgene silencing, genome integrity, and transposon stability (Matzke et al. 2009).

In the RdDM pathway, both 24-nucleotide (nt) siRNAs and long noncoding RNA transcripts are essential for de novo DNA methylation (Wierzbicki et al. 2008). The 24-nt siRNAs are generated in a pathway involving the putative DNA-directed RNA polymerase IV (Pol IV), RDR2 (RNA-dependent RNA polymerase 2), and DCL3 (Dicer-like 3) (Matzke et al. 2009), while noncoding RNAs are produced by another putative DNA-directed RNA polymerase, Pol V (Wierzbicki et al. 2008). The 24-nt siRNAs are loaded onto ARGONAUTE proteins AGO4 and/or AGO6 (Zheng et al. 2007; Matzke et al. 2009) and target genomic regions, possibly by base-pairing with nascent Pol V transcripts from the target loci. The Pol V-dependent RNA transcripts are tethered to AGO4 by the action of KTF1, forming an effector complex containing AGO4, KTF1, Pol V-dependent transcripts, and siRNAs (He et al. 2009a). The effector complex directs DRM2 for de novo methylation of target genomic sequences. The process is facilitated by the chromatin remodeling protein DRD1 and the hinge domain-containing protein DMS3 (Matzke et al. 2009).

RNA Pol IV and Pol V have distinct largest subunitsNRPD1 and NRPE1, respectively-but share some common subunits such as NRPD/E2 and NRPD/E4 (He et al. 2009b). Some subunits of Pol IV and Pol V are also shared with Pol II (Huang et al. 2009; Ream et al. 2009). It appears that the plant-specific RNA Pol IV and Pol V evolved from an ancestral RNA polymerase to function specifically in RdDM. The transcription activity of Pol II is tightly regulated with the help of a series of general transcription factors (Kornberg 2007). Although many general transcription factors of Pol II have been studied extensively, nothing is known about the regulation of transcription by Pol IV and Pol V.

In the present study, we carried out a forward genetic screen for second site suppressors of the DNA demethylase mutant ros1, and identified a transcription factor, RDM4 (RNA-directed DNA Methylation4), that is required for RdDM in Arabidopsis. Interestingly, unlike other RdDM mutants, the rdm4 mutant plants display pleiotropic developmental phenotypes. RDM4 encodes a novel protein that is conserved in eukaryotic organisms. We found that RDM4 interacts with Pol II and Pol V in plants. Our results suggest that RDM4 is a regulator of Pol II and Pol V transcription, and thereby contributes to both development and RdDM.

\section{Results and Discussion}

The rdm4 mutation suppresses transcriptional gene silencing (TGS)

A subset of the RdDM target loci is under dynamic control by DNA methylation and active demethylation (Zhu et al. 2007). In our genetic system, these targets include an active LUCIFERASE transgene driven by the cold and salt stress-responsive RD29A promoter (RD29A$L U C$ ) and the corresponding endogenous RD29A gene, which are silenced when the DNA demethylase gene ROS1 loses its function (Gong et al. 2002). This TGS is caused by DNA hypermethylation, and requires 
He et al.

heterochromatic 24-nt siRNAs from the RD29A promoter (Zheng et al. 2007). The CaMV 35S promoterdriven NPTII transgene (35S-NPTII) linked to the RD29A-LUC transgene is also silenced in ros1 plants (Gong et al. 2002). A T-DNA mutagenized population in the ros1-1 background was screened to identify new components in the RdDM pathway, based on the luminescence phenotype of RD29A-LUC. This screen recovered not only mutants in known RdDM pathway components-such as NRPD1, NRPE1, NRPD2/NRPE2, AGO4, HEN1, DRD1, and HDA6 (He et al. 2009b) - but also several new components. One of the new components is designated as RDM4. The $r d m 4$ mutation released the silencing of the RD29A-LUC transgene in the ros 1 background, resulting in enhanced luminescence after cold or salt treatment of plants (Fig. 1A,B). However, the mutation did not release the silencing of 35S-NPTII in ros1 (Fig. 1C). When the ros $1 \mathrm{rdm} 4$ mutant was crossed to ros1, the F1 plants emitted as little luminescence as the ros 1 plants, suggesting that the $r d m 4$ mutation was recessive. Analysis of the resulting selfed F2 population confirmed the recessive and single-gene nature of the mutation (data not shown).

While the expression of endogenous RD29A, RD29A$L U C$, and $35 S-N P T I I$ was blocked by the ros1 mutation as reported previously (Gong et al. 2002), the rdm4 mutation partially restored the transcript levels of endogenous RD29A and transgene RD29A-LUC, but not of $35 S-N P T I I$ (Fig. 1C). Therefore, the rdm4 mutation releases the silencing of RD29A-LUC but not $35 S$ NPTII. This effect is very similar to that of nrpd1, nrpe1, nrpd2, drd1, and ago6 mutations (Zheng et al. 2007; He et al. 2009b). Because the TGS of the 35S-NPTII transgene is mediated by an siRNA-independent pathway (He et al. 2009b), these results suggest that, like

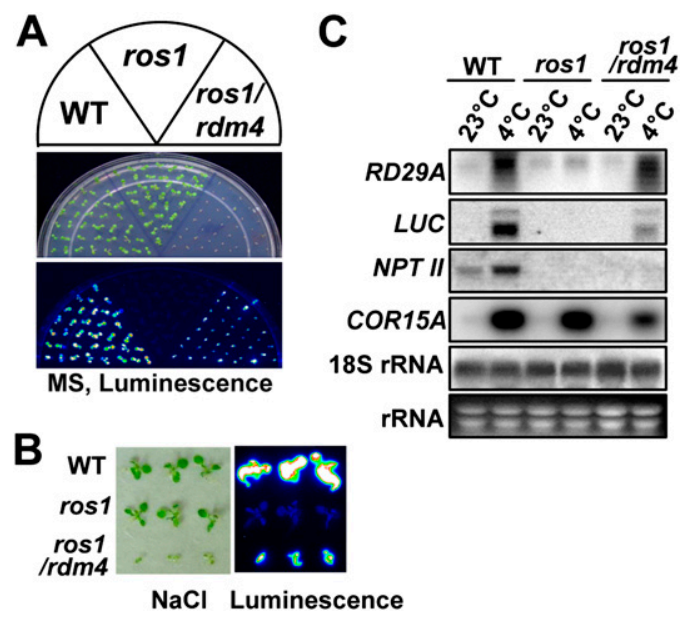

Figure 1. TGS phenotypes of the ros $1 \mathrm{rdm} 4$ mutant plants. $(A, B)$ Expression of the RD29A-LUC transgene by luminescence emission. (A) Wild type, ros1, and ros1rdm4 were grown on MS plates and imaged after cold treatment $\left(24 \mathrm{~h}, 4^{\circ} \mathrm{C}\right) .(B)$ The indicated plants were treated with $200 \mathrm{mM} \mathrm{NaCl}$ for $3 \mathrm{~h}$, followed by luminescence imaging. $(C)$ The transcript levels of endogenous RD29A, and RD29A-LUC and 35S-NPTII transgenes in wild type, ros1, and ros $1 \mathrm{rdm} 4$ were determined by RNA blot analysis. 18S rRNA hybridization and ethidium bromide-stained rRNA bands were used as RNA loading controls, and COR $15 A$ was used as a cold treatment control.
NRPD1, NRPE1, NRPD2, DRD1, and AGO6, RDM4 functions specifically in the siRNA-dependent pathway of TGS.

\section{The rdm4 mutation reduces DNA methylation at RdDM target loci}

To test whether the release of TGS in ros 1 rdm 4 mutant plants correlates with DNA hypomethylation, we determined the DNA methylation status of a 361-basepair (bp) region of the $R D 29 A$ promoter in both the RD29A-LUC transgene and endogenous RD29A by Southern hybridization and bisulfite sequencing. Consistent with previous results, high levels of DNA methylation were observed in ros1 in all three cytosine contexts (CG, CHG, and $\mathrm{CHH}$; $\mathrm{H}$ stands for $\mathrm{A}, \mathrm{T}$, or $\mathrm{C}$ ) at the promoter of both the RD29A-LUC transgene and the endogenous RD29A gene, whereas low levels of DNA methylation were observed in the wild-type plants (Fig. 2A,B). As in ros1nrpd1, the DNA methylation was reduced in ros $1 \mathrm{rdm} 4$ at both the transgene and endogenous RD29A promoters in all cytosine contexts. In both the transgene and endogenous $R D 29 A$ promoters, the reduction in cytosine methylation was dramatic at $\mathrm{CHH}$, modest at $\mathrm{CHG}$, and marginal at CG sites (Fig. $2 \mathrm{~A}, \mathrm{~B})$. For example, at the RD29A-LUC transgene promoter, $\mathrm{CHH}$ methylation was $15.2 \%$ in ros $1,4.4 \%$ in ros $1 \mathrm{rdm} 4$, and $2.8 \%$ in ros $1 \mathrm{nrpd} 1$, while the CG methylation is $75.3 \%, 64.3 \%$, and $58.3 \%$, respectively, for the different genotypes. The inhibitory effect of $\mathrm{rdm} 4$ on DNA methylation was further supported by Southern hybridization. Genomic DNA from wild type, ros1, ros1rdm4, and ros1nrpd1 was digested with a methylation-sensitive restriction enzyme, BstUI (CGCG), followed by Southern hybridization using the RD29A coding region as a probe to assess methylation of the endogenous $R D 29 A$ gene. The result was consistent with the bisulfite sequencing data and showed that the DNA methylation of the endogenous $R D 29 A$ promoter in ros $1 \mathrm{rdm} 4$ and ros1nrpd1 is partially suppressed compared with ros1 (Fig. 2C). Therefore, like nrpd1, rdm4 suppresses TGS of RD29A-LUC and endogenous RD29A in ros 1 by blocking DNA hypermethylation at the $R D 29 A$ promoter.

We assayed the DNA methylation status of the centromeric region using the methylation-sensitive restriction enzyme HaeIII (for $\mathrm{CHH}$ methylation) and the isoschizomers HpaII (for CG and CHG methylation) and MspI (for CG methylation) followed by Southern hybridization. The results showed no differences in DNA methylation of the highly repetitive 180 -bp centromeric repeat among wild-type, ros1, ros $1 \mathrm{rdm} 4$, and ros1nrpd1 mutant plants (Supplemental Fig. S1). The DNA methylation of $5 \mathrm{~S}$ rDNA was also detected by Southern hybridization. In contrast to the heavy methylation of $5 \mathrm{~S}$ rDNA in the wild type and ros1, methylation in all cytosine contexts was clearly reduced in $\operatorname{ros} 1 \mathrm{rdm} 4$, as it was in ros1nrpd1 (Fig. 2D). Southern hybridization also revealed a reduction of DNA methylation at AtMU1 in ros $1 \mathrm{rdm} 4$ and ros1nrpd1, compared with the wild type and ros1 (Fig. 2E). Furthermore, AtSN1 methylation was examined. After digestion with the methylation-sensitive enzyme HaeIII, AtSN1 could be amplified in the wild type and ros1, but not in ros1rdm4, ros1nrpd1, and rdm4 (Fig. 2F), indicating that AtSN1 methylation was blocked by the rdm4 mutation. 

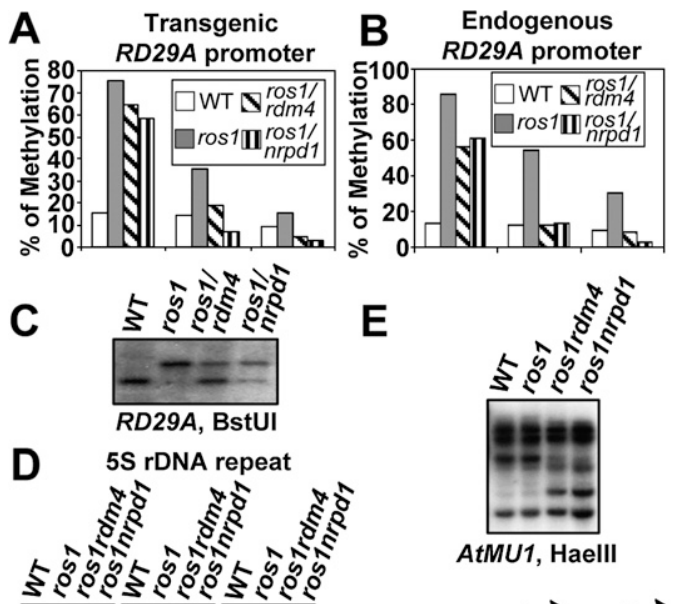

AtMU1, Haell
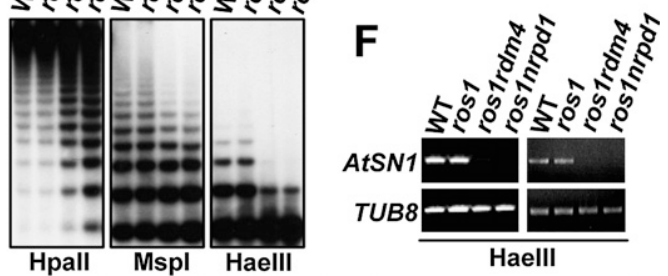

Figure 2. Effect of $r d m 4$ on DNA methylation. The percentage of cytosine methylation at transgene $(A)$ and endogenous $(B) R D 29 A$ promoters was determined by bisulfite sequencing. The percentage of cytosine methylation on $\mathrm{CG}, \mathrm{CHG}$, and $\mathrm{CHH}$ ( $\mathrm{H}$ stands for $\mathrm{A}$, $\mathrm{T}$, or C) sites is shown. (C) The $r d m 4$ mutation reduces DNA methylation at the endogenous $R D 29 \mathrm{~A}$ promoter as determined by Southern hybridization. Genomic DNA was digested with the methylation-sensitive restriction enzyme BstUI. (D) The rdm4 mutation reduces DNA methylation at $5 \mathrm{~S}$ rDNA repeat. $(E)$ Genomic DNA from indicated genotypes was digested with the methylation-sensitive restriction enzyme HaeIII, followed by Southern hybridization with the AtMU1 probe. (F) Effect of $r d m 4$ on DNA methylation of AtSN1. PCR amplification of AtSN1 was performed after the genomic DNA was digested with HaeIII. TUB8 DNA was amplified as an internal control.

The effect of the rdm4 mutation on the expression, siRNAs, and Pol V-dependent transcripts of RdDM target loci

The reduced DNA methylation at the transposons and other RdDM target loci prompted us to examine the expression levels of these loci. We determined the transcript levels of AtSN1, AtGP1, and AtMU1 by semiquantitative RT-PCR. The results indicated that the transcript levels of AtSN1, AtGP1, and AtMU1 were substantially increased in ros $1 \mathrm{rdm} 4, \mathrm{rdm} 4$, and ros1nrpd1, compared with those in the wild type and ros1 (Fig. 3A). The data suggest that, like in ros1nrpd1, the loss of DNA methylation in ros $1 \mathrm{rdm} 4$ and $\mathrm{rdm} 4$ results in increased expression of the RdDM target loci. Previous studies showed that the ROS1 transcript level is down-regulated in RdDM pathway mutants and other mutants with reduced genomic DNA methylation (Huettel et al. 2006; Mathieu et al. 2007). Consistent with the effect of $r d m 4$ on DNA methylation, the ROS1 transcript level was also reduced in ros $1 \mathrm{rdm} 4$ and $\mathrm{rdm} 4$, as well as in ros1nrpd1, compared with the wild type and ros1 (Fig. 3A).

In the ros 1 mutant background, 24-nt siRNAs from the transgene $R D 29 A$ promoter are the trigger of TGS of RD29A-LUC and endogenous RD29A (Gong et al. 2002; Zheng et al. 2007). A small RNA blot analysis showed that 24-nt RD29A promoter siRNAs were reduced in ros 1 rdm4, as they were in ros1nrpe1 (Fig. 3B). In contrast, the siRNAs were completely blocked in ros1nrpd1 (Fig. 3B). We also tested the accumulation of siRNAs from other RdDM target loci, including AtSN1, 5S rDNA/ siRNA1003, AtGP1, AtMU1, Cluster 4, and siRNA02. Similar to the RD29A promoter siRNAs, the accumulation of 24-nt siRNAs from AtSN1, 5S rDNA/siRNA1003, AtGP1, AtMU1, and Cluster 4 was reduced but not abolished in ros1rdm4 (Fig. 3B). siRNA02 was not affected in ros $1 \mathrm{rdm} 4$ (Fig. 3B). The siRNA pattern in ros 1 rdm4 is very similar to that in ros1nrpe1, whereas in ros1nrpd1 the heterochromatic siRNAs were eliminated. The accumulation of microRNA171 (miRNA171) and trans-acting siRNA255 (ta-siRNA255) was also tested, and the results suggest that the $r d m 4$ mutation has little effect on miRNAs and ta-siRNAs (Fig. 3B). Based on these results, we conclude that RDM4 is required for the full accumulation of heterochromatic siRNAs and, like NRPE1 of Pol V, it may also be required for the functioning of the siRNAs through the RdDM pathway.

Pol V was shown recently to generate transcripts from several RdDM target loci, and the nascent transcripts are proposed to function as scaffolds to recruit the AGO4containing RdDM effector complex to the target loci (Wierzbicki et al. 2008). We found that Pol V-dependent transcripts from AtSN1 and IGN5 were reduced in ros $1 \mathrm{rdm} 4$ compared with those in the wild type and ros 1 (Fig. 3C). As expected, the transcripts were blocked in ros1nrpe1, but not much affected in ros1nrpd1 (Fig. 3C).

The effect of the rdm4 mutation on plant development and the expression of Pol II-transcribed genes

The ros $1 \mathrm{rdm} 4$ mutant plants are considerably smaller than the ros 1 or wild-type plants (Fig. 4A). The mutant

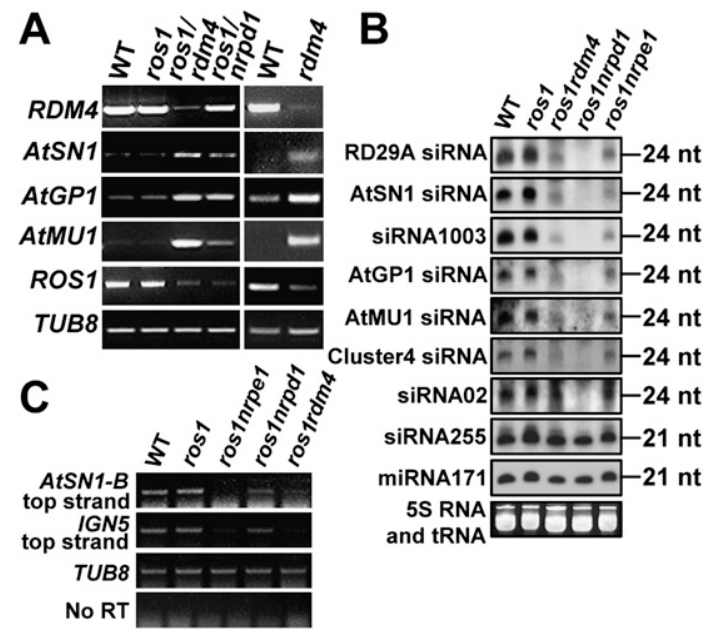

Figure 3. Effect of the $r d m 4$ mutation on RNA transcript levels and siRNA accumulation. $(A)$ The RNA transcript levels of the indicated loci were determined by semiquantitative RT-PCR. TUB8 was used as an internal control. (B) Detection of small RNAs in the indicated genotypes by Northern blot analysis. The ethidium bromide-stained gel corresponding to 5S rRNA and tRNA was included as a loading control. The positions of size markers are indicated ( $24 \mathrm{nt}$ or $21 \mathrm{nt}$ ). (C) RT-PCR detection of Pol V-dependent transcripts from AtSN1-B and IGN5. TUB8 was used as an internal control, and the RNA samples without reverse transcription (No RT) were used as negative control, indicating no DNA contamination. 


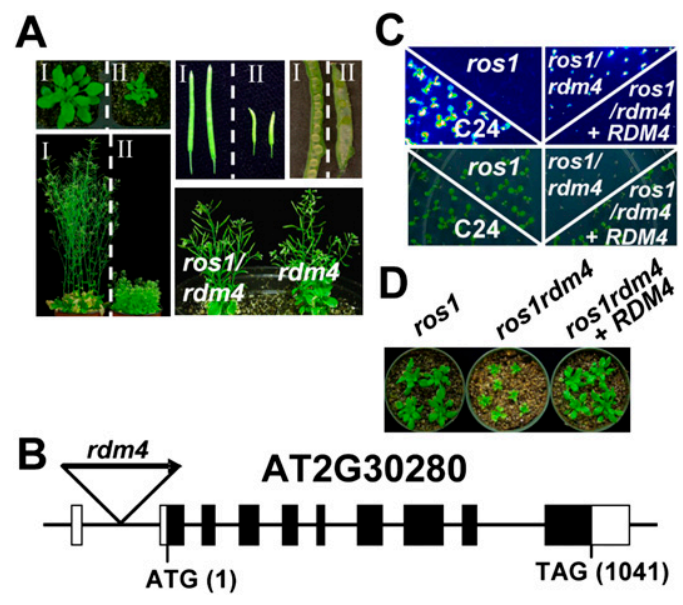

Figure 4. Developmental phenotypes of $r d m 4$ mutant plants and mutant complementation. (A) The pleiotropic developmental phenotypes of ros1rdm4 and $r d m 4$ mutant plants. (Panels I) ros1 plants. (Panels II) ros1rdm4 plants. (B) Diagram of the RDM4 gene showing the position of exons (boxes), introns (lines), and site of T-DNA insertion. (C) The RDM4 genomic construct complements the luminescence phenotype of ros $1 \mathrm{rdm} 4$ in a representative T2 transgenic line. $(D)$ The $R D M 4$ transgene complements the developmental defects of ros1rdm4. The complemented plants shown are T1 transgenic lines.

plants produce shorter siliques and exhibit partial sterility (Fig. 4A). The mutant seeds show a severe dormancy and germinate later than ros1 (Supplemental Fig. S2). Most of the ros1 seeds germinated at the third or fourth day after imbibition, but the ros $1 \mathrm{rdm} 4$ seeds did not all germinate until the 11th day (Supplemental Fig. S2). The developmental phenotypes of ros $1 \mathrm{rdm} 4$ are heritable and recessive, and the phenotypes are also observed in the rdm4 single mutant (Fig. 4A). In a segregating backcrossed F2 population, the morphological phenotypes cosegregated with the luminescence phenotype.

Related to the pleiotropic developmental phenotypes of $r d m 4$, there are changes in the expression of proteincoding genes in the mutant. For example, we noticed that the stress-induced COR15A gene, which was used as a cold stress treatment control for assays on RD29A expression, showed a reduced transcript level in ros $1 \mathrm{rdm} 4$ (Fig. 1C). Microarray analysis showed that, in addition to COR15A, several other abiotic stressresponsive genes-such as KIN1, KIN2, RD26, and $R D 29 B$ - have reduced expression in ros $1 \mathrm{rdm} 4$ compared with ros1 (Supplemental Tables S1, S2). In contrast, many pathogen resistance-related genes-such as $P R 1, P R 4$, $P A D 4$-and several WRKY transcription factors show increased expression in ros1rdm4 compared with ros1, suggesting that the systemic acquired resistance pathway may be activated in the rdm4 mutant (Supplemental Tables S1, S3). The reduced expression of abiotic stressresponsive genes in ros1rdm4 was confirmed by Northern blot analysis and real-time PCR (Supplemental Fig. $\mathrm{S} 3 \mathrm{~A}, \mathrm{~B})$. The cold-induced expression, as well as basal expression, of COR15A and KIN2 was reduced in ros $1 \mathrm{rdm} 4$ compared with that in the wild type and ros1 (Supplemental Fig. S3A,B). Real-time PCR analysis showed that, in contrast to rdm4, the nrpd1 and nrpe1 mutations did not cause a reduction in COR15A or KIN2 expression (Supplemental Fig. S3B). These results suggest that, in addition to its function in RdDM, RDM4 has a role in plant development and in regulating stressresponsive genes, which are presumed to be transcribed by RNA Pol II.

\section{RDM4 encodes a novel protein conserved from yeast to humans}

In the ros $1 \mathrm{rdm} 4$ mutant, a single T-DNA insertion was found in the intron of the $5^{\prime}$-untranslated region (UTR) of AT2G30280 (Fig. 4B). In ros1rdm4 and $r d m 4$, the RDM4 transcript was substantially reduced but not abolished (Fig. 3A), so the rdm4 mutation is a partial loss-offunction allele. To confirm that $R D M 4$ is the correct gene, a construct harboring the full genomic sequence of $R D M 4$ was generated and introduced into ros $1 \mathrm{rdm} 4$ mutant plants. In seven independent $\mathrm{T} 1$ transgenic plants, the wild-type RDM4 transgene rescued the luminescence phenotype of the ros1rdm4 mutant (Supplemental Fig. S4). The DNA methylation status of AtSN1 was tested in wild-type, ros1, ros $1 \mathrm{rdm} 4$, and the seven transgenic T1 plants. The results suggest that the RDM4 transgene also complemented the DNA methylation phenotype of ros1rdm4 (Supplemental Fig. S4). Complementation of the luminescence phenotype was confirmed in T2 transgenic lines (Fig. 4C). Furthermore, the transgene fully rescued the developmental phenotype of the ros1rdm4 mutant (Fig. 4D) and corrected its defects in stress-responsive genes (Supplemental Fig. S3A). Therefore, we conclude that At2G30280 is the RDM4 gene that functions in development and RdDM.

$R D M 4$ encodes a protein of 346 amino acids that has sequence similarity to the yeast IWR1 (interacts with RNA Pol II) and homologous proteins in Drosophila and humans (Supplemental Fig. S5A,B). The homologous proteins in Drosophila and humans have not been characterized. The function of yeast IWR 1 is also unclear, but the protein appears to interact with RNA Pol II and Pol II general transcription factors (Gavin et al. 2006; Krogan et al. 2006). Affinity-capture mass spectrometry experiments identified many Pol II subunits (e.g., RPB2, RPB3, RPB4, RPB5, RPB7, RPB8, RPB9, RPB10, RPB11, RPO21, and RPO26) and several TFIIF subunits (e.g., TFG1 and TFG2) that formed a complex with IWR1. These interactions suggest a role for yeast IWR1 in regulating RNA Pol II transcription. Proteins highly similar to RDM4 can be found in other plant species, such as grapes, rice, and maize (Supplemental Fig. S6A). Phylogenetic analysis shows that RDM4 and its orthologs in grapes, rice, and maize are grouped into a plant-specific clade, which is divided into two subclades: one from dicotyledonous plants, and the other from monocotyledonous plants (Supplemental Fig. S6B). The human IWR1-like protein appears more closely related to the plant RDM4 than to the yeast IWR1 and fly IWR1-like protein (Supplemental Figs. S5A,B, S6B).

\section{RDM4 interacts with RNA Pol II and Pol $V$ in plants}

The RDM4 protein level in ros1rdm4 was substantially reduced compared with that in ros1 (Fig. 5A). The RDM4 protein level was not affected in ros1nrpd1 or ros1nrpe1 (Fig. 5A). Anti-Flag antibodies pulled down RDM4 from extracts of NRPE1-Flag-expressing plants but not NRPD1Flag-expressing plants (Fig. 5B), suggesting that RDM4 interacts with Pol V in vivo. Anti-RDM4 antibodies 
A

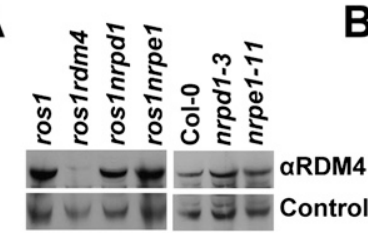

C

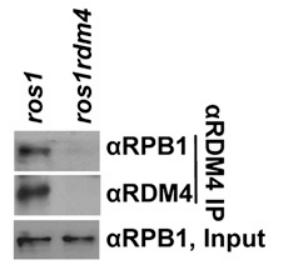

B
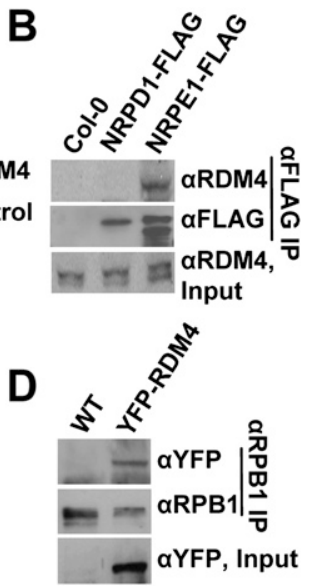

Figure 5. RDM4 interacts with NRPE1 and RPB1. (A) The RDM4 protein level was assessed by Western blot analysis in the indicated genotypes. A nonspecific band (Control) is shown as loading control. (B) Coimmunoprecipitation between RDM4 and NRPE1-Flag. The protein extracts from the indicated genotypes were precipitated by anti-Flag antibody-conjugated beads, and the precipitates were detected by anti-RDM4 and anti-Flag antibodies. $(C)$ Coimmunoprecipitation between RDM4 and RPB1. The protein extracts from ros 1 and $\operatorname{ros} 1 \mathrm{rdm} 4$ were precipitated by anti-RDM4 antibody-conjugated beads, and the precipitates were detected by anti-RPB 1 and antiRDM4 antibodies. $(D)$ The protein extracts from wild-type and YFP$R D M 4$ transgenic plants were precipitated by anti-RPB1 antibodyconjugated beads, and the precipitates were detected by anti-YFP and anti-RPB1 antibodies.

pulled down RPB1 of Pol II in ros1 but not in ros1rdm4 (Fig. 5C). The result suggests an in vivo interaction between RDM4 and Pol II. This is further supported by the observation that antibodies specific for RPB1 could coimmunoprecipitate RDM4-YFP from RDM4-YFPexpressing plants, but not from wild-type control plants (Fig. 5D).

The largest and second largest subunits of RNA Pol IV and Pol V are key components of the RdDM pathway (Matzke et al. 2009). We recently found another subunit, RDM2/NRPD4, that is shared by Pol IV and Pol V, and that is also necessary for the RdDM pathway (He et al. 2009b). Like Pol II (Cramer 2002), Pol IV and Pol V are multisubunit complexes (Huang et al. 2009; Ream et al. 2009). In addition to requiring various polymerase subunits, the functioning of Pol II as well as of Pol I and Pol III requires several positive and negative transcriptional regulators (Wade and Struhl 2008). It is not known whether the functioning of Pol IV and Pol V in RdDM also requires transcriptional regulators and whether such transcriptional regulators are specific for Pol IV and/or Pol $\mathrm{V}$ or are shared with Pol II. RDM4 is possibly the first characterized transcriptional regulator of both Pol II and Pol V. The interaction of yeast IWR1 with Pol II and the general transcription factor TFIIF suggests that IWR1 is a regulator of Pol II transcription (Gavin et al. 2006; Krogan et al. 2006). IWR1 is conserved in yeast, plants, and animals (Supplemental Figs. S5, S6). Our findings of a coimmunoprecipitation between RDM4 and RPB1 and the effect of $r d m 4$ mutation on plant development and on several Pol II-transcribed genes, strongly support that RDM4 is a regulator of Pol II transcription in plants. Our results also demonstrate that RDM4 is required for RdDM and for the accumulation of Pol V transcripts, thereby suggesting a function of RDM4 in regulating Pol $\mathrm{V}$ transcription. The role of RDM4 in RdDM is unlikely to be an indirect effect of its function in Pol II transcription, since the transcript level of none of the RdDM components is affected by the $r d m 4$ mutation (data not shown). Consistent with a direct role of RDM4 in RdDM, we found that RDM4 interacts with Pol V, a key component of the RdDM pathway.

\section{Concluding remarks}

Our results suggest that RDM4 is a transcriptional regulator shared by Pol II and Pol V. Unlike the Pol V-specific nrpe1 mutant or many other RdDM mutants reported to date, which have little or no developmental phenotypes, the $r d m 4$ mutant has strong developmental defects. The pleiotropic phenotypes of $r d m 4$ mutant plants likely reflect a role of RDM4 in Pol II function. Microarray analysis revealed several hundred Pol II-type genes having reduced or increased expression in the rdm4 mutant (Supplemental Table S1), although it is not known which of these genes are directly affected by $r d m 4$. The possible activation of systemic acquired resistance, as suggested by the elevated expression of many pathogen resistancerelated genes in $r d m 4$, may contribute to the slow growth of the mutant plants. Some genes may be particularly sensitive to perturbations of Pol II transcription. Alternatively, RDM4 may specifically regulate the transcription of only a subset of Pol II target genes. In conclusion, our results show that RDM4 functions in both plant development and epigenetic regulation, and suggest that RDM4 may regulate coding genes through Pol II and noncoding loci through Pol V in the RdDM pathway.

\section{Materials and methods}

Plant growth, mutant screening, cloning, and others

A homozygous RD29A-LUC transgene was in the wild-type C24 and ros 1 mutant plants in this study (Ishitani et al. 1997; Gong et al. 2002). A T-DNA mutagenized ros1-1 population was generated as described previously (He et al. 2009b). A 3-d cold stratification was used to promote the uniformity of seed germination. Plants were grown with $16 \mathrm{~h}$ of light and 8 $\mathrm{h}$ of darkness at $23^{\circ} \mathrm{C}$. Based on the expression of the transgenic RD29A promoter-driven luciferase gene by luminescence imaging (Ishitani et al. 1997), putative suppressors of ros1 were identified and transferred to soil. To eliminate false positives, a young leaf taken from each of these seedlings was applied for luminescence imaging after treatment with $200 \mathrm{mM} \mathrm{NaCl}$ for $3 \mathrm{~h}$. Thermal asymmetric interlaced PCR (TAIL-PCR) (Liu et al. 1995) was carried out to determine the T-DNA flanking sequence of the mutants. The full genomic sequence of RDM4 was amplified and cloned into a Gateway vector PMDC164 for Arabidopsis complementation assay. Because ros $1 \mathrm{rdm} 4$ is partially sterile, the F1 plants from the cross of ros $1 \mathrm{rdm} 4$ and $\operatorname{ros} 1$ were used for transformation of the RDM4 complementation construct. Transgenic plants containing the construct in the homozygous ros $1 \mathrm{rdm} 4$ background were identified in the T1 generation of the transformants. Expression of YFP-RDM4 fusion protein was driven by the CaMV 35S promoter. The Fitch-Margoliash method was applied to analyze the protein sequences of yeast IWR1 and IWR1-like proteins in Drosophila, humans, and plants.

\section{RNA analysis}

Northern blot analysis of mRNAs and small RNAs was carried out as described in He et al. (2009b). The sequences of DNA oligos used for probes or probe amplification are listed in Supplemental Table S4.

Semiquantitative RT-PCR was carried out to determine the transcript levels of the RdDM targets, including AtSN1, AtGP1, and AtMU1, as 
He et al.

described in He et al. (2009b). The procedure of RT-PCR amplification of Pol V-dependent transcripts was described by Wierzbicki et al. (2008). Primers used in RT-PCR analysis are listed in Supplemental Table S4.

Microarray analysis was carried out as described previously (Lee et al. 2005) using Affymetrix Arabidopsis ATH1 GeneChip. Two biological replicates were used for each genotype. The preprocessing statistical method RMA (Irizarry et al. 2003) was applied to raw .CEL files for background adjustment and quantile normalization. The log-transformed values were then analyzed using the Bioconductor package siggenes (Tusher et al. 2001). A list of genes with statistically significant changes in expression between the genotypes was generated.

\section{DNA methylation assay}

Bisulfite sequencing, Southern hybridization, and PCR-based DNA methylation assays were carried out as described previously (He et al. 2009a,b).

\section{Coimmunoprecipitation and Western blotting}

Three grams of seedlings from wild-type, NRPD1-Flag, and NRPE1-Flag (driven by their respective endogenous gene promoters) transgenic plants (Pontes et al. 2006) and the other indicated plants were grounded and suspended in $10 \mathrm{~mL}$ of protein extraction buffer. After centrifugation, equal amounts of protein extracts were incubated with the indicated antibody-conjugated beads, followed by washing with protein extraction buffer three times. The precipitated proteins were eluted from the beads with SDS-PAGE sample buffer. The eluted samples were resolved by $12 \%$ SDS-PAGE for Western blotting. The full-length RDM4 ORF was cloned into the bacterial expression vector pET-28a for RDM4 protein production. Anti-RDM4 antibodies were produced by injecting rabbits with purified histine-tagged full-length RDM4 recombinant. The RDM4 antibody was affinity-purified with RDM4 protein-conjugated resin (YenZym Antibodies).

\section{Acknowledgments}

We thank R. Stevenson for technical assistance. This work was supported by National Institutes of Health grants R01GM070795 and R01GM059138 to J.-K.Z.

\section{References}

Chan SW, Henderson IR, Jacobsen SE. 2005. Gardening the genome: DNA methylation in Arabidopsis thaliana. Nat Rev Genet 6: 351-360.

Cramer P. 2002. Multisubunit RNA polymerases. Curr Opin Struct Biol 12: $89-97$.

Gavin AC, Aloy P, Grandi P, Krause R, Boesche M, Marzioch M, Rau C, Jensen LJ, Bastuck S, Dumpelfeld B, et al. 2006. Proteome survey reveals modularity of the yeast cell machinery. Nature 440: 631-636.

Gong Z, Morales-Ruiz T, Ariza RR, Roldan-Arjona T, David L, Zhu JK. 2002. ROS1, a repressor of transcriptional gene silencing in Arabidopsis, encodes a DNA glycosylase/lyase. Cell 111: 803-814.

He XI, Hsu YF, Zhu S, Wierzbicki AT, Pontes O, Pikaard CS, Liu HL Wang CS, Jin H, Zhu JK. 2009a. An effector of RNA-directed DNA methylation in Arabidopsis is an ARGONAUTE 4- and RNA-binding protein. Cell 137: 498-508.

He XJ, Hsu YF, Pontes O, Zhu J, Lu J, Bressan RA, Pikaard C, Wang CS, Zhu JK. 2009b. NRPD4, a protein related to the RPB4 subunit of RNA polymerase II, is a component of RNA polymerase IV and V and is required for RNA-directed DNA methylation. Genes \& Dev 23: 318330.

Huang L, Jones AM, Searle I, Patel K, Vogler H, Hubner NC, Baulcombe DC. 2009. An atypical RNA polymerase involved in RNA silencing shares small subunits with RNA polymerase II. Nat Struct Mol Biol 16: 91-93.

Huettel B, Kanno T, Daxinger L, Aufsatz W, Matzke AJ, Matzke M. 2006. Endogenous targets of RNA-directed DNA methylation and Pol IV in Arabidopsis. EMBO I 25: 2828-2836.

Irizarry RA, Hobbs B, Collin F, Beazer-Barclay YD, Antonellis KJ, Scherf U, Speed TP. 2003. Exploration, normalization, and summaries of high density oligonucleotide array probe level data. Biostatistics 4 249-264.
Ishitani M, Xiong L, Stevenson B, Zhu JK. 1997. Genetic analysis of osmotic and cold stress signal transduction in Arabidopsis: Interactions and convergence of abscisic acid-dependent and abscisic acidindependent pathways. Plant Cell 9: 1935-1949.

Kornberg RD. 2007. The molecular basis of eukaryotic transcription. Proc Natl Acad Sci 104: 12955-12961.

Krogan NJ, Cagney G, Yu H, Zhong G, Guo X, Ignatchenko A, Li J, Pu S, Datta N, Tikuisis AP, et al. 2006. Global landscape of protein complexes in the yeast Saccharomyces cerevisiae. Nature 440: 637643.

Lee BH, Henderson DA, Zhu JK. 2005. The Arabidopsis cold-responsive transcriptome and its regulation by ICE1. Plant Cell 17: 3155-3175.

Liu YG, Mitsukawa N, Oosumi T, Whittier RF. 1995. Efficient isolation and mapping of Arabidopsis thaliana T-DNA insert junctions by thermal asymmetric interlaced PCR. Plant $J$ 8: 457-463.

Mathieu O, Reinders I, Caikovski M, Smathajitt C, Paszkowski J. 2007. Transgenerational stability of the Arabidopsis epigenome is coordinated by CG methylation. Cell 130: 851-862.

Matzke MA, Birchler JA. 2005. RNAi-mediated pathways in the nucleus. Nat Rev Genet 6: 24-35.

Matzke M, Kanno T, Daxinger L, Huettel B, Matzke AJM. 2009. RNAmediated chromatin-based silencing in plants. Curr Opin Cell Biol 21: $367-376$.

Pontes O, Li CF, Nunes PC, Haag J, Ream T, Vitins A, Jacobsen SE, Pikaard CS. 2006. The Arabidopsis chromatin-modifying nuclear siRNA pathway involves a nucleolar RNA processing center. Cell 126: 79-92.

Ream TS, Haag JR, Wierzbicki AT, Nicora CD, Norbeck AD, Zhu JK, Hagen G, Guilfoyle TJ, Pasa-Tolic L, Pikaard CS. 2009. Subunit compositions of the RNA-silencing enzymes Pol IV and Pol V reveal their origins as specialized forms of RNA polymerase II. Mol Cell 33: 192-203.

Tusher VG, Tibshirani R, Chu G. 2001. Significance analysis of microarrays applied to the ionizing radiation response. Proc Natl Acad Sci 98: 5116-5121.

Wade JT, Struhl K. 2008. The transition from transcriptional initiation to elongation. Curr Opin Genet Dev 18: 130-136.

Wassenegger M, Heimes S, Riedel L, Sanger HL. 1994. RNA-directed de novo methylation of genomic sequences in plants. Cell 76: 567-576.

Wierzbicki AT, Haag JR, Pikaard CS. 2008. Noncoding transcription by RNA polymerase $\mathrm{Pol} \mathrm{IVb} / \mathrm{Pol} \mathrm{V}$ mediates transcriptional silencing of overlapping and adjacent genes. Cell 135: 635-648.

Zheng X, Zhu J, Kapoor A, Zhu JK. 2007. Role of Arabidopsis AGO6 in siRNA accumulation, DNA methylation and transcriptional gene silencing. EMBO I 26: 1691-1701.

Zhu J, Kapoor A, Sridhar VV, Agius F, Zhu JK. 2007. The DNA glycosylase/lyase ROS1 functions in pruning DNA methylation patterns in Arabidopsis. Curr Biol 17: 54-59. 


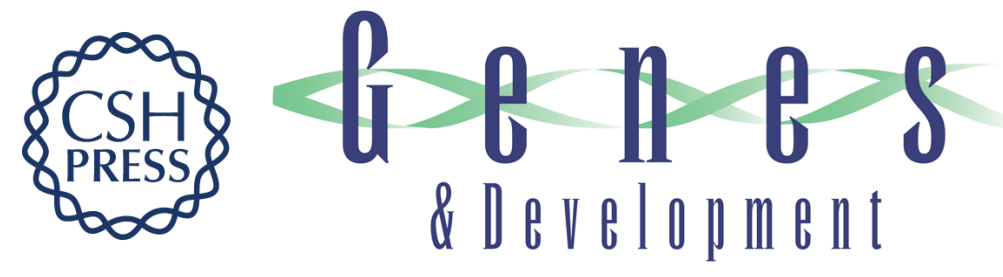

\section{A conserved transcriptional regulator is required for RNA-directed DNA methylation and plant development}

Xin-Jian He, Yi-Feng Hsu, Shihua Zhu, et al.

Genes Dev. 2009, 23: originally published online November 10, 2009

Access the most recent version at doi:10.1101/gad.1851809

\section{Supplemental http://genesdev.cshlp.org/content/suppl/2009/10/05/gad.1851809.DC1 Material}

References This article cites 25 articles, 5 of which can be accessed free at: http://genesdev.cshlp.org/content/23/23/2717.full.html\#ref-list-1

\section{License}

Email Alerting

Service

Receive free email alerts when new articles cite this article - sign up in the box at the top right corner of the article or click here.

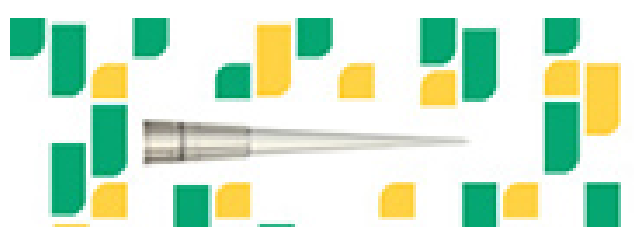

Focused on your science. 